\title{
Research Paper: Ibuprofen Protection Against Restrained Chronic Stress-induced Depression in Male Rats
}

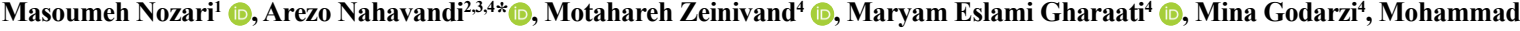 \\ Ahmadi ${ }^{3}$, Nida Jamali-Raeufy ${ }^{4}$ (1)
}

1. Neuroscience Research Center, Institute of Neuropharmacology, Kerman University of Medical Sciences, Kerman, Iran

2. Department of Neuroscience, Faculty of Advanced Technologies in Medicine, Iran University of Medical Sciences, Tehran, Iran.

3. Neuroscience Research Center, Iran University of Medical Sciences, Tehran, Iran.

4. Department of Physiology, School of Medicine, Iran University of Medical Sciences, Tehran, Iran.

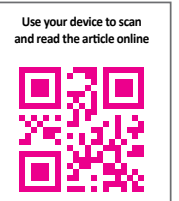

Citation: Nozari, M., Nahavandi, A., Zeinivand, M., Eslami Gharaati, M., Godarzi, M., \& Ahmadi, M., et al. Ibuprofen Protection Against Restrained Chronic Stress-induced Depression in Male Rats. Basic and Clinical Neuroscience, 11(4), 413-422. http:// dx.doi.org/10.32598/ben.11.4.1775.2

http://dx.doi.org/10.32598/ben.11.4.1775.2

\section{Article info:}

Received: 08 Apr 2019

First Revision: 10 May 2019

Accepted: 15 Oct 2019

Available Online: $01 \mathrm{Jul} 2020$

Keywords:

Depression, Ibuprofen, Passive avoidance, IL-6, Forced swimming test

\begin{abstract}
A B S T RA C T
Introduction: Stress predisposes organisms to depression and cognitive impairments, and seems to interact with metabolic homeostasis. The inflammatory response and the upregulation of proinflammatory cytokines are some of the consequences related to chronic stress. In this study, we investigated the preventive effect of chronic administration of ibuprofen, as an inhibitor of cyclooxygenases, on the cognitive and behavioral alterations and the weight gain reduction induced by simultaneous chronic restraint stress in rats.

Materials and Methods: Male Wistar rats were subjected to chronic restraint stress and injected daily with the variable doses of ibuprofen or vehicle, for 21 consecutive days. Then, all animals were tested with the forced swim test and passive avoidance conditioning. Also, the weight of the animals was recorded before and after the interventions. Ultimately, plasma interleukin 6 (IL-6) levels were measured.

Results: Chronic stress increased depressive-like behaviors, impaired learning, and disrupted the normal weight gain. However, the animals that received the highest dose of ibuprofen showed less depressive-like behaviors, a better avoidance memory, and a higher weight gain. However, the level of plasma IL-6 did not differ significantly between the study groups.

Conclusion: The administration of ibuprofen prevents the cognitive and behavioral consequences of chronic stress. During the recovery, the plasma levels of IL-6 were not elevated by stress, and the IL-6 levels did not predict the behavioral performance of the stressed animals. The exact mechanisms of the protective effects of ibuprofen against chronic stress need to be further investigated.
\end{abstract}




\section{Highlights}

- Stress causes depression and cognitive impairments.

- Stress brings about inflammatory and the upregulation of proinflammatory.

- Ibuprofen as an inhibitor of cyclooxygenases and preventive effect of chronic administration.

\section{Plain Language Summary}

Stress causes depression and cognitive impairments, and seems to interact with metabolic homeostasis. It causes inflammatory response and the upregulation of proinflammatory. The reciprocal link between the immune and central nervous systems plays a key role in stress-induced neuroinflammation and depression. We think ibuprofen prevent the stress-induced behavioral, cognitive, and weight alterations following a chronic restraint stress model in rats. The highest dose of ibuprofen showed less depressive-like behaviors. Forced Swimming Test (FST) to measure depressive-like behaviors was used. Finally, the plasma level of interleukin 6 (IL-6) was measured, because the IL-6 is an indicator of inflammation in depressed patients.

\section{Introduction}

tress is defined as an imbalance in homeostasis. Every unknown new situation may activate the stress response that modulates the homeostasis. The stress response is the organism's intrinsic reaction to physically or psychologically disturbing factors (Garcia-Bueno et al., 2008). Short-term stress reactions have evolved as adaptive strategies to foster survival (Buchanan, 2000; Miller \& Raison, 2016). However, long-term ongoing stress may lead to mental and physical health problems (Munhoz et al., 2008). Therefore, neuropsychiatric conditions are expected to follow long-lasting chronic stress (Rivat et al., 2010). Repeated, unpredictable, and chronic stress is the critical trigger factor for depression (Hammen, 2005; Grippo \& Scotti, 2013) and inflammatory response, within the brain and periphery (Garate et al., 2013; Grippo \& Scotti, 2013; Raison \& Miller, 2013). The reciprocal link between the immune and central nervous systems plays a key role in stress-induced neuroinflammation and depression (Grippo \& Scotti, 2013). The upregulation of Cyclooxygenase $2(\mathrm{COX}-2)$ is proposed as one of the underlying mechanisms of stress-induced depression (Munhoz et al., 2008; Garate et al., 2013). Nonsteroidal Anti-Inflammatory Drugs (NSAIDs) are agents that exert the anti-inflammatory and analgesic effects through the inhibition of the COX (Rao \& Knaus, 2008). Although the efficacy of the NSAIDs as an antidepressive and anti-inflammatory adjuvant (Köhler, Petersen, Mors, \& Gasse, 2015) is well established, the protective potential of NSAIDs against chronic stress has partially become clear.
Administering the ibuprofen (as a prominent NSAID and nonselective cyclooxygenase inhibitor), we aimed to prevent the stress-induced behavioral, cognitive, and weight alterations following a chronic restraint stress model in rats. In the present study, the passive avoidance learning task and the shuttle box were used as an indicator of stress-induced cognitive impairments. Also, we used the Forced Swimming Test (FST) to measure depressive-like behaviors. Finally, the plasma level of interleukin 6 (IL-6) was measured, because the IL-6 is an indicator of inflammation in depressed patients; the plasma level of IL-6 increases after the chronic stress test in rodents (Mehrpouya et al., 2015).

\section{Materials \& Methods}

\subsection{Animals and treatments}

A total number of 40 adult male Wistar rats with the weight range of 200 to $250 \mathrm{~g}$, and the age range of 7 to 9 weeks (Pasteur Institute of Iran, Karaj, Iran) were kept under the standard light-dark cycle $(12 \mathrm{~h}: 12 \mathrm{~h})$, temperature $\left(21^{\circ} \mathrm{C}-25^{\circ} \mathrm{C}\right)$, and humidity $(45-55 \%)$ with free access to food and water. All handling procedures complied with the international guidelines for the care and use of laboratory animals (EU Directive 2010/63/ EU for animal experiments) and approved by the Ethics Committee of Iran University of Medical Sciences. Also, the experiments complied with the ARRIVE (Animal Research: Reporting of In Vivo Experiments) guidelines for reporting experiments involving animals.

For behavioral experiments, all animals were transported to the test room, one hour before the test. Using a 
Table 1. Treatments and group sizes (n: 8)

\begin{tabular}{cc}
\hline Treatment & Abbreviation \\
\hline Sham & Control \\
\hline Chronic restraint stress & CRS \\
\hline CRS and ibuprofen $60 \mathrm{mg} / \mathrm{kg}$ & CRS+lbu-60 \\
\hline CRS and ibuprofen $30 \mathrm{mg} / \mathrm{kg}$ & CRS+lbu-30 \\
\hline CRS and ibuprofen $15 \mathrm{mg} / \mathrm{kg}$ & CRS+lbu-15 \\
\hline
\end{tabular}

NEUR SCIENCE

completely randomized design, the animals were categorized. They received sham (non-stress+vehicle), or stress and the intraperitoneal injection of ibuprofen (Ibuprofen; Damavand Darou, Tehran, Iran) in the volume of 15, 30, or $60 \mathrm{mg}$ per each kilogram of body weight. Ibuprofen was dissolved in distilled water before the injection (de la Puente et al., 2015). The doses were based on previous studies (Zaminelli et al., 2014). Table 1 summarizes the treatments for each group of the study animals. (As the sign and symptoms were not responsive to 15 and $30 \mathrm{mg} /$ $\mathrm{kg}$ of ibuprofen, we didn’t mention them later on.)

\subsection{Experimental procedures}

As illustrated in Figure 1, the animals were weighed before any handling (on day 0 ). The animals of the stress group were daily injected with ibuprofen or vehicle for 21 consecutive days (day 1-21). Also, 30 minutes after each injection, they were restrained for 6 hours $(8 \mathrm{AM}$ to 2 PM) with the standard rat Plexiglas restrainers (Campos, Fogaça, Aguiar, \& Guimaraes, 2013). While the restrained rats were completely immobile with no access to food and water, the rats of the sham group were not subjected to stress and returned to their home cages after the vehicle injection. On day 23, the animals were trained for passive avoidance in the shuttle box and tested on day 24 .

On day 28, the subjects were habituated for the FST that was performed on day 29. Ultimately, on day 36 , the animals were weighed and deeply anesthetized with ketamine. The blood samples were taken for the assessments of IL-6 and corticosterone plasma levels with the Enzyme-Linked Immunosorbent Assay (ELISA) method. The animals were euthanized afterward. All the ex- periments were carried out by two observers who were blinded to the treatments.

\subsection{Passive Avoidance Memory test}

The custom-built shuttle box was made of two wooden compartments (each, $30 \times 20 \times 20 \mathrm{~cm}$ ) separated by a guillotine door $(20 \times 15 \mathrm{~cm})$. One of the boxes was equipped with a $20 \mathrm{~W}$ lamp, but the other one was dark and had a grid floor ( $3 \mathrm{~mm}$ diameter, $10 \mathrm{~mm}$ distance). For habituation, the animals were placed in the bright part and the guillotine door was opened after five seconds. Once the animal passed through the door, it was closed and the animal was returned to the home cage, after 20 seconds. Thirty minutes later, one trial training session was performed similar to the habituation procedure, but once the animal entered the dark compartment, it received a footshock $(50 \mathrm{~Hz}, 1 \mathrm{~mA}, 3 \mathrm{~s})$. Then, the animal was returned to the home cage. On the next day, the animal was placed in the bright compartment and the guillotine door was opened after 20 seconds. The latency time to enter the dark compartment was measured with a stopwatch; the cut off time was 300 seconds (Hosseinzadeh, Roshan, \& Pourasghar, 2013; Barzegar et sl., 2015).

\subsection{Forced Swimming test}

Pretest: The animals were individually placed at a depth of $18 \mathrm{~cm}$ in a transparent cylinder $(80 \mathrm{~cm}$ height, $30 \mathrm{~cm}$ diameter) filled with $24^{\circ} \mathrm{C}$ water, for 15 minutes. Then, they were dried with a towel and returned to their cages.

Swim test: At the same conditions as the pretest, the animals were placed in the cylinder, and the video was recorded. After five minutes, they were dried and returned to their

\begin{tabular}{|c|c|c|c|c|c|c|c|}
\hline \multirow{2}{*}{$\begin{array}{l}\text { Weinghing } \\
\text { animals }\end{array}$} & \multirow{2}{*}{$\begin{array}{l}\text { Stressinduction } \\
\text { and injections }\end{array}$} & \multicolumn{2}{|c|}{ Passive avoidance } & \multirow{2}{*}{ Recovery period } & \multicolumn{2}{|c|}{ Forced swim test } & \multirow{2}{*}{$\begin{array}{l}\text { Weighing animals } \\
\text { Blood sampling } \\
\text { and sacrifice }\end{array}$} \\
\hline & & Training & Retention & & Habituation & Test & \\
\hline Day 0 & Day 1-21 & Day 23 & Day 24 & Day 25-27 & Day 28 & Day 29 & Day 36 \\
\hline
\end{tabular}

Figure 1. Experimental procedure 
cages. Cumulative immobility duration was measured by two observers who were blinded to the treatments (Cryan, Valentino, \& Lucki, 2005; Slattery \& Cryan, 2012).

\subsection{IL-6 ELISA}

The animals were recovered for a week to avoid transient increments in the IL-6 levels because of the behavioral experiments. Next, the rats were anesthetized with ketamine $(100 \mathrm{mg} / \mathrm{kg})$ and xylazine $(10 \mathrm{mg} / \mathrm{kg})$, then, the heart apex was exposed and blood samples (1 $\mathrm{mL}$ ) were taken. The samples were centrifuged (10 min, $664 \times \mathrm{g}$ ), and the plasma was stored at $-20^{\circ} \mathrm{C}$. The plasma IL-6 levels were quantified using commercially available ELISA kit (eBioscience, Vienna, Austria), according to manufacturer's instructions.

\subsection{Corticosterone ELISA}

The rat blood samples were centrifuged at $664 \times \mathrm{g}$ for 10 minutes. The sera were immediately separated and stored at $-80 \mathrm{oC}$ until the examination. The corticosterone level was measured using the ELISA kit (Zellbio GmbH, Germany), according to the manufacturer's protocol.

\subsection{Transmission electron microscopy}

Two rats of each group were anesthetized with the intraperitoneal injection of ketamine-xylazine combination (10:1) and perfused intracardially with $0.9 \%$ sodium

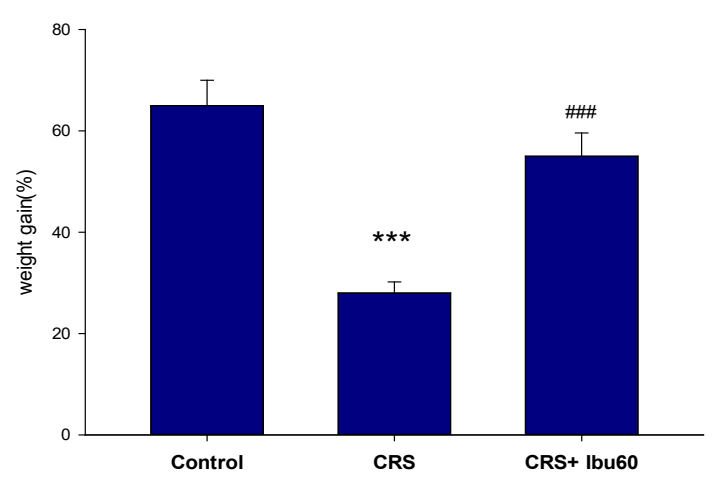

NEUR SCIENCE

Figure 2. Ibuprofen treatment facilitating weight gain in chronically stressed rats

The results of the one-way ANOVA and the Tukey post hoc test $(n=8)$ revealed that the animals in the CRS group gained significantly lower weight than the control group. Also, the CRS+Ibu-60 group gained significantly more weight than the CRS group;

*** $\mathrm{P}<0.001$;

\#\#\# $\mathrm{P}<0.001$ chloride, 10X phosphate-buffered saline, and 4\% paraformaldehyde. Then, they were decapitated and their brains removed. After tissue processing, $5-\mu \mathrm{m}$ paraffinembedded sections were provided. These structures become visualized in the electron microscope at the magnifications range of 50 to 1000000 times. The protocols given hereafter can be used for light microscopy and transmission electron microscopy.

\subsection{Statistical analysis}

Values were expressed as Mean \pm Standard deviation. The SigmaPlot-14 (Systat Software Inc.) was used to analyze the obtained data. To analyze variations among three or more groups, the one-way analysis of variance followed by the Tukey test for post hoc analysis was performed. The $\mathrm{P}$-value of less than $0.05(\mathrm{P}<0.05)$ was considered statistically significant.

\section{Results}

\subsection{Ibuprofen protects against stress-induced} weight loss

According to Figure 2, chronic stress disturbs normal weight gain, and ibuprofen is preventive against these changes. The stress group had the smallest weight gain, while the CRS+Ibu-60 group showed a high mean of weight gain. The CRS+Ibu-60 group had significantly

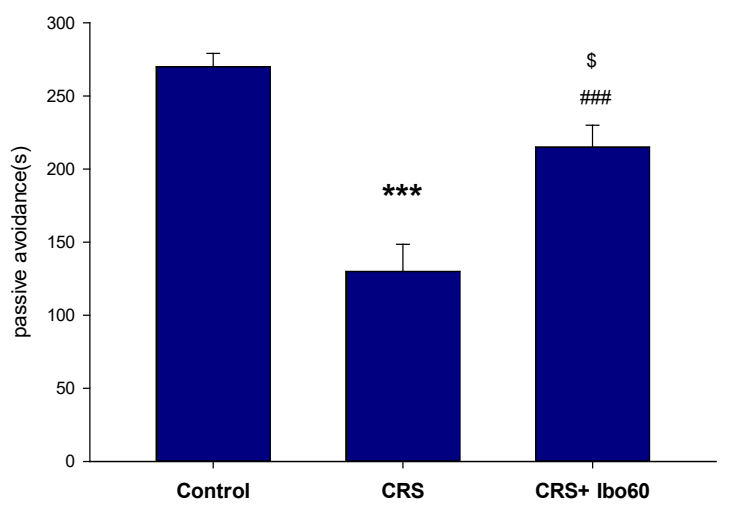

NEUR SCIENCE

Figure 3. Ibuprofen improving passive avoidance learning in chronically stressed rats

The results of the one-way ANOVA and the Tukey post hoc test $(n=8)$ revealed that the animals in the CRS group gained significantly lower memory function scores than the control group. Also, the CRS+Ibu-60 group had significantly better avoidance memory function, compared with the control and CRS groups;

* $\mathrm{P}<0.05$

*** $\mathrm{P}<0.001$ 


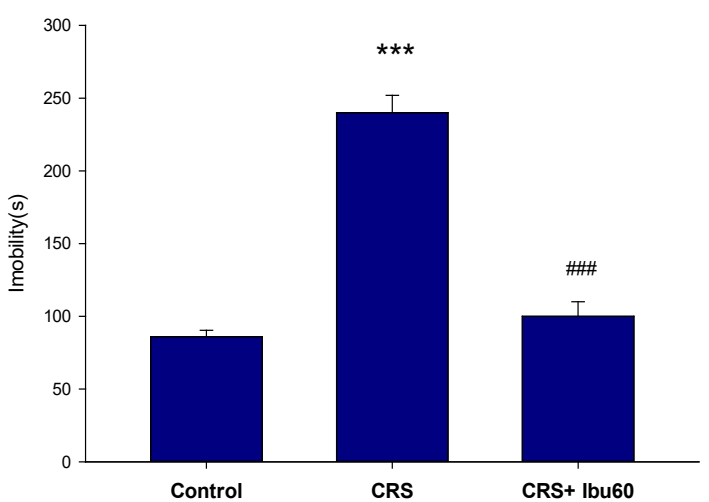

NEUR SCIENCE

Figure 4. Ibuprofen protection against stress-induced depressive-like immobility in FST

The results of the one-way ANOVA and the Tukey post hoc test $(n=8)$ revealed that the animals in the CRS group had significantly higher immobility time than the control group. Also, the CRS+Ibu-60 group had significantly shorter immobility during the FST, compared with the CRS group;

*** $\mathrm{P}<0.001$

\#\#\# $\mathrm{P}<0.001$

higher weight gain, compared with the stress group $\left(\mathrm{F}_{6,35}=\right.$ $6.46, \mathrm{P}<0.001)$.

\subsection{Ibuprofen improves stress-induced learning} deficits

Chronic stress impairs learning processes and negatively impacts learning avoidance (Chida, Sudo, Mori, \& Kubo, 2006; Radahmadi, Alaei, Sharifi, \& Hosseini, 2013). Here, chronically stressed animals that were treated with a high dose of Ibuprofen $(60 \mathrm{mg} / \mathrm{kg})$ significantly improved avoidance performance. Compared with the control $(\mathrm{P}<0.05)$ and CRS $(\mathrm{P}<0.001)$ groups, the $\mathrm{CRS}+\mathrm{Ibu}-60$ group had significantly $\left(\mathrm{F}_{6,35}=15.60, \mathrm{P}<0.001\right)$ better performance in avoidance memory (Figure 3 ).

3.3. Ibuprofen prevents depressive-like immobility in the FST

The FST is one of the most frequently used tests for both the assessments of depressive-like behavior in rodents and the antidepressant-like properties of the pharmacological agents (Cryan, Valentino, \& Lucki, 2005; Slattery \& Cryan, 2012). Compared with the control group (217.83 $\pm 25.40 \mathrm{~s})$, the CRS group showed a higher immobility time $(86.50 \pm 5.49 \mathrm{~s}, \mathrm{P}<0.001)$. However, the

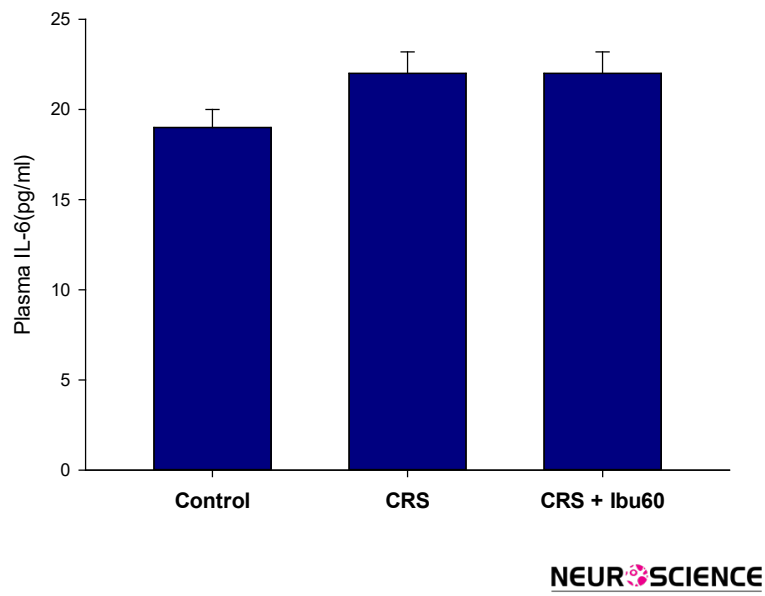

Figure 5. Plasma IL-6 level in chronically stressed animals during recovery

The results of the one-way ANOVA and the Tukey post hoc test $(n=8)$ revealed that the study groups did not significantly differ in the IL-6 levels $(\mathrm{P}=0.559)$.

CRS+Ibu-60 group $(100.33 \pm 9.95 \mathrm{~s})$ had a significantly $\left(\mathrm{F}_{6,35}=36.38, \mathrm{P}<0.001\right)$ shorter immobility time, compared with the CRS group (Figure 4).

\subsection{Plasma IL-6 level}

The one-way ANOVA did not indicate any significant difference in the plasma levels of IL- 6 between the study groups $\left(\mathrm{F}_{6,35}=0.824, \mathrm{P}=0.559\right.$; Figure 5).

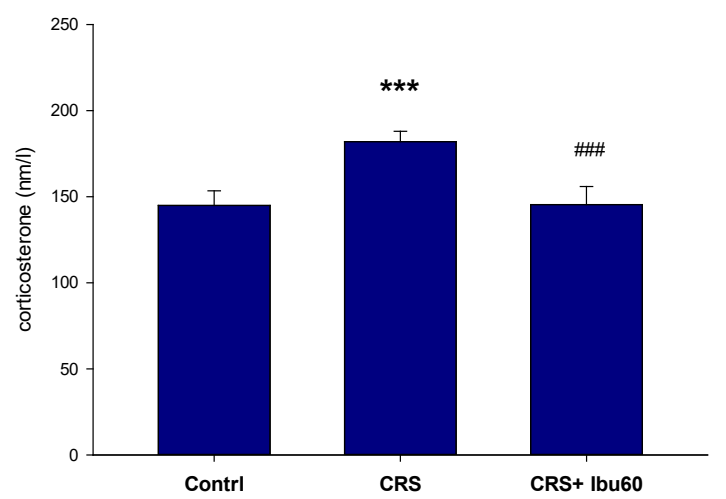

NEUR SCIENCE

Figure 6. Ibuprofen improving the lower level of corticosterone in chronically stressed animals

The results of the one-way ANOVA and the Tukey post hoc test $(n=8)$ revealed that the animals in the CRS group had significantly higher corticosterone levels than the control group. Also, the CRS+Ibu-60 group had significantly lower corticosterone levels compared with the CRS group;

$* * * \mathrm{P}<0.001$

$\# \# P<0.001$ 


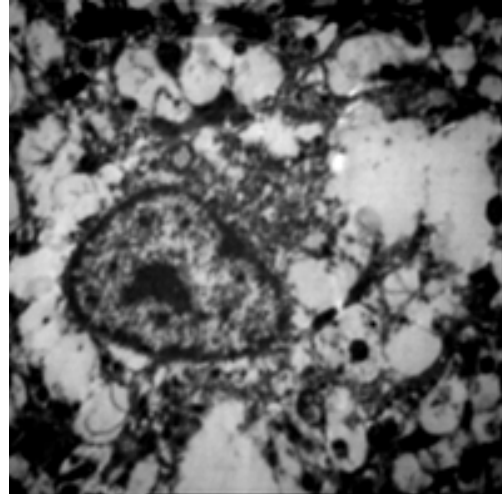

Control

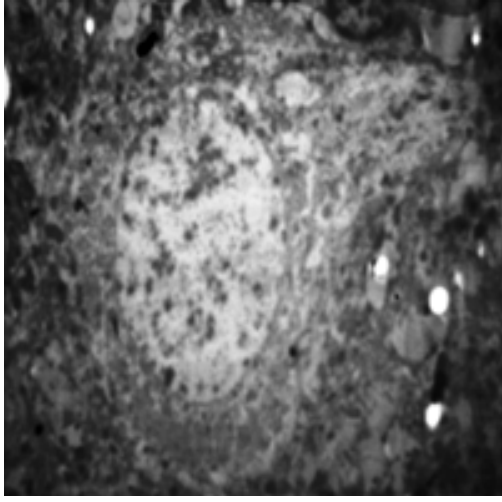

CRS

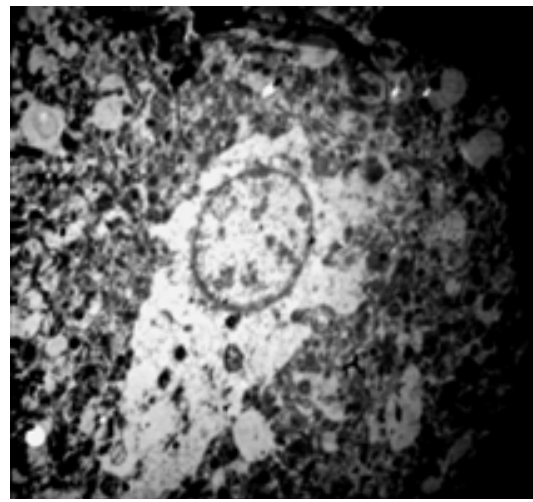

CRS+Ibu-60

NEUR SCIENCE

Figure 7. Ibuprofen improving apoptotic changes in chronically stressed animals

Electron microscopy imaging showed that the animals in the CRS group had significantly higher apoptotic changes than the control group. Also, the CRS+Ibu-60 group had significantly lower apoptotic changes, compared with the CRS group. The images were at an original magnification of $\times 5000$. The data were compared using the one-way ANOVA and the Tukey post hoc test $(\mathrm{n}=8)$.

\subsection{Plasma corticosterone level}

Compared with the control group $(217.83 \pm 25.40 \mathrm{~s})$, the CRS group had a significantly higher plasma corticosterone level $(86.50 \pm 5.49 \mathrm{~s}, \mathrm{P}<0.001)$. However, the corticosterone plasma level of the CRS+Ibu-60 group $(100.33 \pm 9.95 \mathrm{~s})$ was significantly $\left(\mathrm{F}_{6,35}=33.38, \mathrm{P}<0.001\right)$ lower than the CRS group (Figure 6).

3.6. Ibuprofen protects against stress-induced apoptotic changes

The transmission electron microscopy results of the CA1 region showed apoptotic changes, including the chromatin margination and dissolution in the cell nucleus of stressed rats. However, we found the large and round nuclei and the intracellular organelles within the cytoplasm of both the control and CRS+Ibu-60 groups $\left(\mathrm{F}_{6,35}=36.27, \mathrm{P}<0.001\right.$; Figure 7$)$.

\section{Discussion}

The present study is the first research investigating the preventive effect of ibuprofen on chronic stress-induced depressive-like behavior in rats. Our results provided a better insight into the stress-induced depressive-like behaviors and examined the potential of ibuprofen as a preventive medication during chronic stress. In the present study, a dose-dependent manner of ibuprofen administration showed that the highest dose of ibuprofen $(60 \mathrm{mg})$ had the best efficiency, and prevented all the stress-induced symptoms. However, the moderate dose
(30 mg) just improved the immobility behavior during the FST. Also, the lowest dose of ibuprofen $(15 \mathrm{mg})$ had no detectable effect on any measurements. Therefore, we focused on the highest dose of ibuprofen $(60 \mathrm{mg} / \mathrm{kg})$ in the following. It should be emphasized that ibuprofen plays an anti-inflammatory role in higher doses, which is seen over the weeks following the administration.

Ibuprofen plays a role both as an anti-inflammatory and a neuroprotective agent in conditions, such as Parkinson and Alzheimer diseases (Wilkinson et al., 2012). Also, chronic ibuprofen administration can reverse depressive behavior in the Parkinson disease model of the rats (Zaminelli et al., 2014). Restraint-induced stress results in oxidative stress, inflammation, and depressive-like symptoms (Gárate et al., 2013). Ibuprofen as a non-specific COX inhibitor may protect against restraint-induced chronic stress via the blockage of this process (Mancuso et al., 2007). In response to stressors, there is a fundamental, dynamic, and reciprocal relationship between the corticosterone and the immune system (Bowers, Bilbo, Dhabhar, \& Nelson, 2008). Following the stressors, pro-inflammatory cytokines, such as tumor necrosis factor- $\alpha$ increase, and result in the overactivity of the HPA axis. The increased corticosterone would suppress the high levels of inflammatory modulators (Leakey et al., 1994). Consequently, both the corticosterone and the inflammatory modulators rise (Besedovsky et al., 1991).

The normal body weight gain of the rats was reduced by the CRS paradigm; this result is in line with the pre- 
vious reports about the impaired weight gain induced by depression following the CRS (Christiansen, Olesen, Wörtwein, \& Woldbye, 2011). Chronic restraint stress increases the activity of the HPA axis and induces the signs and symptoms of depression, such as decreased appetite and reduced body weight gain (Kaestner et al., 2005).

In the present study, we found an increased immobility behavior in the CRS group, compared with the control and CRS+Ibu-60 groups. Immobility behavior could be translated into behavioral despair (Cryan \& Mombereau, 2004). The restraint-induced chronic stress and depression result in the various modifications of the brain, including decreased dopamine level and reduced hippocampal volume and plasticity, which are almost the result of neuroinflammation (Lee et al., 2013).

Abnormal HPA axis activity is correlated with stress-related conditions, such as mental depression (Chang et al., 2009). In such conditions, the HPA activity is usually exaggerated (Chang et al., 2009). As mentioned earlier, the activity of the inflammatory modulators increased HPA activity. It seems that the high dose of ibuprofen has interrupted this loop and decreased behavioral despair. The high dose of ibuprofen could improve the passive avoidance task in the rat depression model. Hippocampus is the main organ involved in learning and memory and is susceptible to inflammation, apoptosis, and damage following the mental depression. The hippocampus function is best evaluated by the passive avoidance task (Ho, Sommers, \& Lucki, 2013). Our findings indicated a normal passive avoidance task in the CRS+Ibu-60 group, compared with the control group. Observing a functional hippocampus in the rats of both intact group and CRS+Ibu-60 group indicates the preventive effect of Ibu 60 on hippocampal damage in stressful situations.

We found no significant increase in plasma IL-6 levels in our stress-induced model. Explaining this result, it is suggested that the time of sampling has been overlapped with the decrement of IL- 6 by the first surge of corticosterone. The short course of stress induction (21 days) in our model may be another possible reason. It seems that the rats required more duration and the time of the CST to develop high levels of IL-6. Several studies have shown that chronic neuro-inflammatory and depressive-like behavior are essential for the elevation of plasma IL-6 (Voorhees et al., 2013). Another study confirmed the IL-6 increase following 28 days of restraint-induced stress in mice (Biesmans et al., 2015). It seems that 21 days of restraint stress is not an appropriate protocol for the IL-6 studies. Possibly, if we continued this phase, we could see the plasma IL-6 increment. The threeweek protocol carried out in the present study explains why we could not find a significant increase in IL-6.
The electron microscope findings showed the signs of apoptotic changes in the CA1 area of the CRS group. Mental depression as a neuro-inflammatory process is associated with apoptosis, and the hippocampus is one of the most susceptible organs for such changes (Zunszain, Anacker, Cattaneo, Carvalho, \& Pariante, 2011). Although there are reports about the apoptotic effect of ibuprofen in various conditions, a study revealed that all the NSAIDs, including ibuprofen, inhibit the nitric oxideinduced apoptosis in a non-cyclooxygenase-dependent manner in chondrocytes (Borutaite \& Brown, 2005). The NSAIDs inhibit the nitric oxide-induced apoptosis and the differentiation of articular chondrocytes independent of cyclooxygenase activity (Borutaite \& Brown, 2005).

Currently, there are controversial discussions regarding the efficacy of NSAIDs against depression. In the present study, we did not investigate the efficiency of ibuprofen as an antidepressant-like drug, but we rather tried to determine the protective value of chronic ibuprofen administration against depression during a chronic restrained stress period. Based on our results, the chronic and simultaneous administration of ibuprofen during stress dose-dependently prevents the metabolic, cognitive, and behavioral consequences of the chronic restraint stress in rats.

\section{Ethical Considerations}

\section{Compliance with ethical guidelines}

All ethical principles were considered in this article.

\section{Funding}

This research did not receive any specific grant from funding agencies in the public, commercial, or not-for-profit sectors.

\section{Authors' contributions}

All authors contributed equally in preparing all parts of the research.

\section{Conflict of interest}

The authors declared no conflict of interest. 


\section{References}

Barzegar, S., Komaki, A., Shahidi, S., Sarihi, A., Mirazi, N., \& Salehi, I. (2015). Effects of cannabinoid and glutamate receptor antagonists and their interactions on learning and memory in male rats. Pharmacology Biochemistry and Behavior, 131, 87-90. [DOI:10.1016/j.pbb.2015.02.005] [PMID]

Besedovsky, H. O., Del Rey, A., Klusman, I., Furukawa, H., Arditi, G. M., \& Kabiersch, A. (1991). Cytokines as modulators of the hypothalamus-pituitary-adrenal axis. The Journal of Steroid Biochemistry and Molecular Biology, 40(4-6), 613-8. [DOI:10.1016/09600760(91)90284-C]

Biesmans, S., Bouwknecht, J. A., Ver Donck, L., Langlois, X., Acton, P. D., \& De Haes, P., et al. (2015). Peripheral administration of tumor necrosis factor-alpha induces neuroinflammation and sickness but not depressive-like behavior in mice. BioMed Research International, 2015. [DOI:10.1155/2015/716920] [PMID] [PMCID]

Borutaite, V. \& Brown, G. (2005). What else has to happen for nitric oxide to induce cell death? Portland Press Limited, 716920 http:/ / downloads.hindawi.com/journals/bmri/2015/716920.pdf

Bowers, S. L., Bilbo, S. D., Dhabhar, F. S., \& Nelson, R. J. (2008) Stressor-specific alterations in corticosterone and immune responses in mice. Brain Behavior E Immunity, 22(1), 105-13. [DOI:10.1016/j.bbi.2007.07.012] [PMID] [PMCID]

Buchanan, K. L. (2000). Stress and the evolution of conditiondependent signals. Trends in Ecology E Evolution, 15(4), 156-60. [DOI:10.1016/S0169-5347(99)01812-1]

Campos, A. C., Fogaça, M. V., Aguiar, D. C., \& Guimaraes, F. S. (2013). Animal models of anxiety disorders and stress. Revista Brasileira de Psiquiatria, 35(2), S101-11. [DOI:10.1590/1516-44462013-1139] [PMID]

Chang, L., Sundaresh, S., Elliott, J., Anton, P. A., Baldi, P., \& Licudine, A. (2009). Dysregulation of the Hypothalamic-Pituitary-Adrenal (HPA) axis in irritable bowel syndrome. Neurogastroenterology $\mathcal{E}$ Motility 21(2), 149-59. [DOI:10.1111/j.1365-2982.2008.01171.x] [PMID] [PMCID]

Chida, Y., Sudo, N., Mori, J., \& Kubo, C. (2006). Social isolation stress impairs passive avoidance learning in Senescence-Accelerated Mouse (SAM). Brain Research, 1067(1), 201-8. [DOI:10.1016/j. brainres.2005.10.042] [PMID]

Christiansen, S. H., Olesen, M. V., Wörtwein, G., \& Woldbye, D. P. D. (2011). Fluoxetine reverts chronic restraint stress-induced depression-like behaviour and increases neuropeptide $\mathrm{Y}$ and galanin expression in mice. Behavioural Brain Research, 216(2), 585 91. [DOI:10.1016/j.bbr.2010.08.044] [PMID]

Cryan, J. F., \& Mombereau, C. (2004). In search of a depressed mouse: utility of models for studying depression-related behavior in genetically modified mice. Molecular Psychiatry, 9(4), 326-7. [DOI:10.1038/sj.mp.4001457] [PMID]

Cryan, J. F., Valentino, R. J., \& Lucki, I. (2005). Assessing substrates underlying the behavioral effects of antidepressants using the modified rat forced swimming test. Neuroscience $\mathcal{E}$ Biobehavioral Reviews, 29(4-5), 547-69. [DOI:10.1016/j.neubiorev.2005.03.008] [PMID]

de la Puente, B., Romero-Alejo, E., Vela, J. M., Merlos, M., Zamanillo, D., \& Portillo-Salido, E. (2015). Changes in saccharin preference behavior as a primary outcome to evaluate pain and analgesia in acetic acid-induced visceral pain in mice. Journal of Pain Research, 8, 663-73. [DOI:10.2147/JPR.S91230] [PMID] [PMCID]
Gárate, I., Garcia-Bueno, B., Madrigal, J. L. M., Caso, J. R., Alou, L. \& Gomez-Lus, M. L. et al. (2013). Stress-induced neuroinflammation: role of the Toll-like receptor-4 pathway. Biological Psychiatry, 73(1), 32-43. [DOI:10.1016/j.biopsych.2012.07.005] [PMID]

Gárate, I., Garcia-Bueno, B., Madrigal, J. L. M., Caso, J. R., Alou, L. Gomez-Lus, M. L., et al. (2013). Stress-induced neuroinflammation: Role of the Toll-like receptor-4 pathway. Biological Psychiatry, 73(1), 32-43. [DOI:10.1016/j.biopsych.2012.07.005] [PMID]

García-Bueno, B., Caso, J. R., \& Leza, J. C. (2008). Stress as a neuroinflammatory condition in brain: Damaging and protective mechanisms. Neuroscience E Biobehavioral Reviews, 32(6), 1136-51. [DOI:10.1016/j.neubiorev.2008.04.001] [PMID]

Grippo, A. J., Scotti M. A. (2013). Stress and neuroinflammation. Modern Trends in Pharmacopsychiatry, 28, 20-32. [DOI:10.1159/000343965] [PMID]

Hammen, C. (2005). Stress and depression. Annual Review of Clinical Psychology, 1, 293-319. [DOI:10.1146/annurev. clinpsy.1.102803.143938] [PMID]

Ho, N., Sommers, M. S., \& Lucki, I. (2013). Effects of diabetes on hippocampal neurogenesis: links to cognition and depression. Neuroscience E Biobehavioral Reviews, 37(8), 1346-62. [DOI:10.1016/j. neubiorev.2013.03.010] [PMID] [PMCID]

Hosseinzadeh, S., Roshan, V. D., \& Pourasghar, M. (2013). Effects of intermittent aerobic training on passive avoidance test (shuttle box) and stress markers in the dorsal hippocampus of wistar rats exposed to administration of homocysteine. Iranian Journal of Psychiatry and Behavioral Sciences, 7(1), 37-44. [PMCID] [PMID]

Kaestner, F., Hettich, M., Peters, M., Sibrowski, W., Hetzel, G., \& Ponath, G., et al. (2005). Different activation patterns of proinflammatory cytokines in melancholic and non-melancholic major depression are associated with HPA axis activity. Journal of Affective Disorders, 87(2-3), 305-11. [DOI:10.1016/j.jad.2005.03.012] [PMID]

Köhler, O., Petersen, L., Mors, O., \& Gasse, C. (2015). Inflammation and depression: combined use of selective serotonin reuptake inhibitors and NSAIDs or paracetamol and psychiatric outcomes. Brain and Behavior, 5(8), e00338. [DOI:10.1002/brb3.338] [PMID] [PMCID]

Leakey, J. E., Chen, S. H. U., Manjgaladze, M., Turturro, A., Duffy, P. H., Pipkin, J. L., \& Hart, R. W. (1994). Role of Glucocorticoids and "Caloric Stress" in Modulating the Effects of Caloric Restriction in Rodents a. Annals of the New York Academy of Sciences, 719(1), 171-94. [DOI:10.1111/j.1749-6632.1994.tb56828.x] [PMID]

Lee, B., Sur, B., Park, J., Kim, S. H., Kwon, S., \& Yeom, M., et al (2013). Chronic administration of baicalein decreases depression-like behavior induced by repeated restraint stress in rats. The Korean Journal Of Physiology \& Pharmacology, 17(5), 393-403. [DOI:10.4196/kjpp.2013.17.5.393] [PMID] [PMCID]

Mancuso, C., Scapagini, G., Curro, D., Giuffrida Stella, A. M., De Marco, C., \& Butterfield, D. A., et al. (2007). Mitochondrial dysfunction, free radical generation and cellular stress response in neurodegenerative disorders. Front Biosci, 12(1), 1107-23. [DOI:10.2741/2130] [PMID]

Mehrpouya, S., Nahavandi, A., Khojasteh, F., Soleimani, M., Ahmadi, M., \& Barati, M. (2015). Iron administration prevents BDNF decrease and depressive-like behavior following chronic stress. Brain Research, 1596, 79-87. [DOI:10.1016/j.brainres.2014.10.057] [PMID] 
Miller, A. H., \& Raison, C. L. (2016). The role of inflammation in depression: From evolutionary imperative to modern treatment target. Nature Reviews Immunology, 16(1), 22. [DOI:10.1038/nri.2015.5] [PMID] [PMCID]

Munhoz, C. D., Garcia-Bueno, B., Madrigal, J. L. M., Lepsch, L. B., Scavone, C., \& Leza, J. C. (2008). Stress-induced neuroinflammation: mechanisms and new pharmacological targets. Brazilian Journal of Medical and Biological Research, 41(12), 1037-46. [DOI:10.1590/S0100-879X2008001200001] [PMID]

Radahmadi, M., Alaei, H., Sharifi, M. R., \& Hosseini, N. (2013). The effect of synchronized running activity with chronic stress on passive avoidance learning and body weight in rats. International Journal of Preventive Medicine, 4(4), 430-7. [DOI:10.5812/asjsm.34532] [PMID] [PMCID]

Raison, C. L., \& Miller, A. H. (2013). Role of inflammation in depression: implications for phenomenology, pathophysiology and treatment. In Inflammation in Psychiatry, 28, 33-48. [DOI:10.1159/000343966] [PMID]

Rao, P., \& Knaus, E. E. (2008). Evolution of Nonsteroidal Anti-inflammatory Drugs (NSAIDs): Cyclooxygenase (COX) inhibition and beyond. Journal of Pharmacy \& Pharmaceutical Sciences, 11(2), 81s-110s. [DOI:10.18433/J3T886] [PMID]

Rivat, C., Becker, C., Blugeot, A., Zeau, B., Mauborgne, A., Pohl, M., \& Benoliel, J. J. (2010). Chronic stress induces transient spinal neuroinflammation, triggering sensory hypersensitivity and long-lasting anxiety-induced hyperalgesia. Pain, 150(2), 358-68. [DOI:10.1016/j.pain.2010.05.031] [PMID]

Slattery, D. A., \& Cryan, J. F. (2012). Using the rat forced swim test to assess antidepressant-like activity in rodents. $\mathrm{Na}$ ture Protocols, 7(6), 1009-014. [DOI:10.1038/nprot.2012.044] [PMID]

Voorhees, J. L., Tarr, A. J., Wohleb, E. S., Godbout, J. P., Mo, X., \& Sheridan, J. F., et al. (2013). Prolonged restraint stress increases IL-6, reduces IL-10, and causes persistent depressivelike behavior that is reversed by recombinant IL-10. PloS One, 8(3), e58488. [DOI:10.1371/journal.pone.0058488] [PMID] [PMCID]

Wilkinson, B. L., Cramer, P. E., Varvel, N. H., Reed-Geaghan E., Jiang, Q., \& Szabo, A., et al. (2012). Ibuprofen attenuates oxidative damage through NOX2 inhibition in Alzheimer's disease. Neurobiology of Aging, 33(1), 197-e21. [DOI:10.1016/j. neurobiolaging.2010.06.014] [PMID] [PMCID]

Zaminelli, T., Gradowski, R. W., Bassani, T. B., Barbiero, J. K., Santiago, R. M., \& Maria-Ferreira, D., et al. (2014). Antidepressant and antioxidative effect of ibuprofen in the rotenone model of Parkinson's disease. Neurotoxicity Research, 26(4) 351-62. [DOI:10.1007/s12640-014-9467-y] [PMID]

Zunszain, P. A., Anacker, C., Cattaneo, A., Carvalho, L. A., \& Pariante, C. M. (2011). Glucocorticoids, cytokines and brain abnormalities in depression. Progress in Neuro-Psychopharmacology and Biological Psychiatry, 35(3), 722-9. [DOI:10.1016/j. pnpbp.2010.04.011] [PMID] [PMCID] 
This Page Intentionally Left Blank 